\title{
Internal-wave reflection from uniform slopes: higher harmonics and Coriolis effects
}

\author{
T. Gerkema \\ Royal Netherlands Institute for Sea Research, P.O. Box 59, 1790 AB Den Burg, The Netherlands
}

Received: 20 March 2006 - Revised: 15 May 2006 - Accepted: 15 May 2006 - Published: 6 July 2006

\begin{abstract}
Weakly nonlinear reflection of internal waves from uniform slopes produces higher harmonics and mean fields; the expressions are here derived for constant stratification and with Coriolis effects fully included, i.e. the horizontal component $\tilde{f}$ of the earth rotation vector (referred to as "non-traditional") is taken into account. Uniformity in one of the horizontal directions is assumed. It is shown that solutions can be as readily derived with as without $\tilde{f}$; hence there is no need to make the so-called Traditional Approximation. Examples of reflecting internal-wave beams are presented for super-inertial, inertial and sub-inertial frequencies. The problem of resonant and non-resonant forcing of the second harmonic is studied for single plane waves; unlike under the Traditional Approximation, the problem of reflection from a horizontal bottom no longer forms a singular case. Non-traditional effects are favourable to resonant forcing at near-tidal rather than near-inertial frequencies, and generally increase the intensity of the second harmonic. Strong stratification tends to suppress non-traditional effects, but a neartotal suppression is only attained for high values of stratification that are characteristic of the seasonal thermocline; in most parts of the ocean, non-traditional effects can therefore be expected to be important.
\end{abstract}

\section{Introduction}

Interaction of monochromatic internal waves can produce internal waves whose frequencies are double, triple etc. that of the primary wave; they are called higher harmonics. A straightforward example of interaction is when an internal wave reflects from a (sloping) bottom: nonlinear effects are then at work at the junction of the incident and reflected waves. This mechanism of generation was previously ana-

Correspondence to: T. Gerkema

(gerk@nioz.nl) lyzed for single plane internal waves in a rotating stably stratified fluid by Thorpe (1987), and for general internal-wave forms (including beams), but without rotation, by Tabaei et al. (2005). In the former case, gravity and the Coriolis force act as restoring forces; in the latter case, only gravity. A vivid illustration of second harmonics, generated by reflecting internal waves, was recently produced in laboratory experiments (Peacock and Tabaei, 2005); experiments on near-critical reflection are presented by Gostiaux et al. (2006). Numerical experiments on internal tides too provide examples of generation of higher harmonics by reflecting beams (Lamb, 2004; Gerkema et al., 2006a). These results are, qualitatively at least, supported by internal-wave spectra deduced from oceanic observations (van Haren et al., 2002), in which higher harmonics of the semi-diurnal internal tide were found. We note that these spectra contain also a strong peak at the inertial frequency, which however does not seem to be accompanied by higher harmonics; we put forward a possible explanation in Sect. 6.3.

In the above-mentioned studies, the rotation vector (if included at all) was assumed to be parallel to gravity, hence vertical. This amounts to making the so-called Traditional Approximation. If one abandons this approximation, a horizontal component $\tilde{f}=2 \Omega \cos \phi$ ( $\Omega$ the earth angular velocity, $\phi$ latitude) comes into play, which produces additional terms in the Coriolis force. This component was recently shown to be important for low-frequency internal waves in weakly stratified fluids (Gerkema and Shrira, 2005). Since the deep ocean, where much of the internal-wave reflection takes place, is indeed weakly stratified (i.e. the buoyancy frequency $N$ is not much higher than $\Omega$ ), we include the horizontal component in the present study, thereby generalizing the earlier studies on internal-wave reflection. One of its effects is to increase the range of allowable internal-wave frequencies (see, e.g., LeBlond and Mysak, 1978). In particular, internal waves can now exist at sub-inertial frequencies (i.e. wave frequency $\sigma<|f|$, with $f=2 \Omega \sin \phi$ ). An example of

Published by Copernicus GmbH on behalf of the European Geosciences Union and the American Geophysical Union. 
second harmonics generated by reflecting sub-inertial waves will be given in Sect. 6.2. Another effect is the change in steepness at which energy propagates. This steepness moreover depends on the horizontal orientation of the waves in the geographical plane. It will be shown that this anisotropy affects also the generation of second harmonics, for example the loci at which resonant forcing occurs (Sect. 6.3).

It was already pointed out by Baines and Miles (2000) that the component $\tilde{f}$ (hereafter referred to as "non-traditional") can be easily included in internal-tide generation problems. The same conclusion was reached by Gerkema and Shrira (2005) regarding the linear theory of internal-wave propagation in general. This will now be taken one step further, into nonlinear internal-wave dynamics, specifically the generation of higher harmonics. We proceed by using an expansion similar to the one used by Tabaei et al. (2005). The principal differences from their study are that we include Coriolis effects (in their full form, moreover), and that we introduce the bottom slope (Sect. 5) only after we have first solved the general problem (Sects. 2 to 4); this actually simplifies the derivation. Examples and an analysis of non-traditional effects, with a view to applications in the deep ocean, are then given in Sect. 6.

\section{Basic equations}

We adopt a coordinate system in which the $x$-axis is rotated by an angle $v$ (anticlockwise) with respect to the west-east direction, and define the Coriolis parameters

$\left(f_{c}, f_{s}, f\right)=2 \Omega(\cos v \cos \phi, \sin v \cos \phi, \sin \phi)$.

We assume latitude $\phi$ to be fixed ( $f$-plane). The components $f_{c}$ and $f_{s}$ are proportional to $\tilde{f}$ (i.e. to cosine of latitude), and would both be absent under the Traditional Approximation. In this coordinate system, the nonlinear equations in an incompressible fluid read (LeBlond and Mysak, 1978):

$$
\begin{aligned}
u_{t}+(\boldsymbol{u} \cdot \nabla) u-f v+f_{c} w & =-p_{x} \\
v_{t}+(\boldsymbol{u} \cdot \nabla) v+f u-f_{s} w & =-p_{y} \\
w_{t}+(\boldsymbol{u} \cdot \nabla) w+f_{s} v-f_{c} u & =-p_{z}+b \\
u_{x}+v_{y}+w_{z} & =0 \\
b_{t}+(\boldsymbol{u} \cdot \nabla) b+N^{2} w & =0,
\end{aligned}
$$

where $u, v$ and $w$ are the velocity components in the $x, y$ and $z$ direction, respectively; $p$ is the departure of pressure from its hydrostatic value (divided by a constant reference density, $\left.\rho_{*}\right) ; b$ is the buoyancy, i.e. $-g \rho / \rho_{*}$, where $\rho$ is the departure of density from its hydrostatic value $\rho_{0}(z)$. Hereafter we assume $N^{2}=-\left(g / \rho_{*}\right) d \rho_{0} / d z$ to be constant.

We consider plane waves travelling in the $x$-direction, so that $\partial / \partial y=0$. This allows us to introduce a stream function $\psi\left(u=\psi_{z}, w=-\psi_{x}\right)$, in terms of which Eqs. (1)-(5) become

$\nabla^{2} \psi_{t}+J\left(\nabla^{2} \psi, \psi\right)-f_{s} v_{x}-f v_{z}+b_{x}=0$

$$
\begin{array}{r}
v_{t}+J(v, \psi)+f_{s} \psi_{x}+f \psi_{z}=0 \\
b_{t}+J(b, \psi)-N^{2} \psi_{x}=0,
\end{array}
$$

with the Jacobian defined as $J(a, b)=a_{x} b_{z}-a_{z} b_{x}$, and $\nabla^{2}=\partial_{x}^{2}+\partial_{z}^{2}$. Note that the terms involving $f_{c}$ cancelled. Non-traditional effects, now present only via $f_{s}$, are strongest if $x$ (i.e. wave-energy propagation) is meridional $(\nu= \pm \pi / 2)$. For zonal propagation $(\nu=0, \pi)$, on the other hand, non-traditional effects are absent from Eqs. (6)-(8) (but not from Eqs. 1 and 3!).

\section{Expansion}

We consider weakly nonlinear waves, monochromatic (with frequency $\sigma$ ) at lowest order, and write the fields in a formal expansion in which $\epsilon$, a measure of the intensity of the wave, serves as the small parameter:

$$
\begin{aligned}
& \psi=\epsilon\{\Psi \exp (-i \sigma t)+\text { c.c. }\}+\epsilon^{2}\left\{\Psi_{0}+\left[\Psi_{2} \exp (-2 i \sigma t)+\text { c.c. }\right]\right\}+\cdots \\
& v=\epsilon\{V \exp (-i \sigma t)+\text { c.c. }\}+\epsilon^{2}\left\{V_{0}+\left[V_{2} \exp (-2 i \sigma t)+\text { c.c. }\right]\right\}+\cdots
\end{aligned}
$$

$b=\epsilon\{\Gamma \exp (-i \sigma t)+$ c.c. $\}+\epsilon^{2}\left\{\Gamma_{0}+\left[\Gamma_{2} \exp (-2 i \sigma t)+\right.\right.$ c.c. $\left.]\right\}+\cdots$

where we anticipate that mean fields (with index 0 ) and second harmonics (with index 2) will appear at order $\epsilon^{2}$; the former is time-independent, the latter has frequency $2 \sigma$. Note that no complex conjugates are added to the mean fields; they will be found to be real by themselves.

We follow the procedure of Tabaei et al. (2005), but with Coriolis effects now included. A further distinction is that we first derive the general solution, irrespective of the presence of topography. In principle, one can continue the expansion (9)-(11) to any order (note, however, the caveat mentioned at the end of Sect. 4.2), but we will only consider the problem up to order $\epsilon^{2}$.

\section{General solution}

For later convenience, we introduce abbreviations $A_{n}, B$ and $C_{n}$ for the coefficients of the governing partial differential equations, to be found at subsequent orders, as well as the corresponding characteristic coordinates $\xi_{n}$ and $\eta_{n}$ :

$$
\begin{aligned}
& A_{n}=N^{2}-(n \sigma)^{2}+f_{s}^{2} ; \quad B=f f_{s} ; \quad C_{n}=f^{2}-(n \sigma)^{2} \\
& \mu_{n, \pm}=\frac{B \pm\left(B^{2}-A_{n} C_{n}\right)^{1 / 2}}{A_{n}} \\
& \xi_{n}=\mu_{n,+} x-z ; \quad \eta_{n}=\mu_{n,-} x-z,
\end{aligned}
$$

where it will be understood that the subscript $n$ is dropped if $n=1$ (i.e. at $\operatorname{order} \epsilon$ ). 


\subsection{Order $\epsilon$; primary wave}

By substituting Eqs. (9)-(11) into Eqs. (6)-(8), we obtain, at lowest order,

$$
\begin{aligned}
-i \sigma \nabla^{2} \Psi-\left(f_{s} \partial_{x}+f \partial_{z}\right) V+\Gamma_{x} & =0 \\
-i \sigma V+\left(f_{s} \partial_{x}+f \partial_{z}\right) \Psi & =0 \\
-i \sigma \Gamma-N^{2} \Psi_{x} & =0
\end{aligned}
$$

implying

$A \Psi_{x x}+2 B \Psi_{x z}+C \Psi_{z z}=0$,

as obtained earlier by Gerkema and Shrira (2005). (Note that the term with the mixed derivative would disappear under the Traditional Approximation, $B=0$.) In terms of the characteristic coordinates $\xi$ and $\eta$, Eq. (18) becomes $\Psi_{\xi \eta}=0$, whose general solution is

$\Psi=F(\xi)+G(\eta)$,

for arbitrary functions $F$ and $G$, each describing propagation of wave-energy along one of the two characteristic coordinates. The other fields can be expressed in terms of $\Psi$ as

$\Gamma=\frac{i N^{2}}{\sigma} \Psi_{x} ; \quad V=-\frac{i}{\sigma}\left(f_{s} \Psi_{x}+f \Psi_{z}\right)$.

With this, the lowest-order problem has been fully solved.

\subsection{Order $\epsilon^{2}$; mean field}

Gathering the time-independent terms resulting from the substitution of Eqs. (9)-(11) in Eqs. (6)-(8), we find

$$
\begin{array}{r}
{\left[J\left(\nabla^{2} \Psi, \Psi^{*}\right)+\text { c.c. }\right]-\left(f_{s} \partial_{x}+f \partial_{z}\right) V_{0}+\Gamma_{0, x}=0} \\
{\left[J\left(V, \Psi^{*}\right)+\text { c.c. }\right]+\left(f_{s} \partial_{x}+f \partial_{z}\right) \Psi_{0}=0} \\
{\left[J\left(\Gamma, \Psi^{*}\right)+\text { c.c. }\right]-N^{2} \Psi_{0, x}=0 .}
\end{array}
$$

We can use Eq. (20) to rewrite the Jacobian in Eq. (23), and then use the general identity $i J\left(P_{x}, P^{*}\right)+$ c.c. $=i J\left(P, P^{*}\right)_{x}$ to obtain

$\Psi_{0}=\frac{i}{\sigma} J\left(\Psi, \Psi^{*}\right)$.

Specifically, substitution of the lowest-order general solution (19) yields

$\Psi_{0}=\frac{2}{\sigma}\left(\mu_{+}-\mu_{-}\right) \operatorname{Im}\left[F^{\prime}(\xi) G^{\prime}(\eta)^{*}\right]$.

(Primes denote derivatives with respect to the characteristic coordinates.) This expression confirms the well-known fact that no nonlinear contributions arise from an interaction of one plane internal wave ( $F$, say) with itself; only junctions of plane waves having different directions provide such terms.

Equation (22) is now automatically satisfied, too. The remaining fields $V_{0}$ and $\Gamma_{0}$ cannot be uniquely determined because of geostropic degeneracy, as is clear from Eq. (21). This is a common problem in rectification studies; it can be resolved either by adding friction, or by changing the setting to an initial-value problem (Visser, 1994). We do not pursue this point further, because the residual fields play no role in finding the second harmonics, the primary aspect of this study. (The geostropic fields would however start to play a role at order $\epsilon^{3}$, and from then on solutions involve an indeterminacy.)

\subsection{Order $\epsilon^{2}$; second harmonic}

Gathering the second harmonics $\exp (-2 i \sigma t)$ resulting from the substitution of Eqs. (9)-(11) in Eqs. (6)-(8), we find

$$
\begin{array}{r}
-2 i \sigma \nabla^{2} \Psi_{2}+J\left(\nabla^{2} \Psi, \Psi\right)-\left(f_{s} \partial_{x}+f \partial_{z}\right) V_{2}+\Gamma_{2, x}=0 \\
-2 i \sigma V_{2}+J(V, \Psi)+\left(f_{s} \partial_{x}+f \partial_{z}\right) \Psi_{2}=0 \\
-2 i \sigma \Gamma_{2}+J(\Gamma, \Psi)-N^{2} \Psi_{2, x}=0
\end{array}
$$

implying

$$
A_{2} \Psi_{2, x x}+2 B \Psi_{2, x z}+C_{2} \Psi_{2, z z}=2 i \sigma J\left(\nabla^{2} \Psi, \Psi\right)-\left(f_{s} \partial_{x}+f \partial_{z}\right) J(V, \Psi)+J(\Gamma, \Psi)_{x} .
$$

The last two terms on the right-hand side can be simplified using the lowest-order Eqs. (15)-(17), giving

$$
A_{2} \Psi_{2, x x}+2 B \Psi_{2, x z}+C_{2} \Psi_{2, z z}=3 i \sigma J\left(\nabla^{2} \Psi, \Psi\right) .
$$

The general solution to this equation reads

$\Psi_{2}=F_{2}\left(\xi_{2}\right)+G_{2}\left(\eta_{2}\right)+\Psi_{2, p}$,

where $F_{2}$ and $G_{2}$ are arbitrary functions, representing solutions in the absence of forcing; $\xi_{2}$ and $\eta_{2}$ are the characteristic coordinates defined in Eq. (14). The forcing, on the right-hand side of Eq. (29), is taken into account via $\Psi_{2, p}$, a particular solution to Eq. (29); an expression for $\Psi_{2, p}$ is derived below.

For later reference, we note that the right-hand side of Eq. (29) can be written in a more explicit form by using the lowest-order general solution (19):

$$
J\left(\nabla^{2} \Psi, \Psi\right)=-\left(\mu_{+}-\mu_{-}\right)\left[\left(1+\mu_{+}^{2}\right) F^{\prime \prime \prime}(\xi) G^{\prime}(\eta)-\left(1+\mu_{-}^{2}\right) G^{\prime \prime \prime}(\eta) F^{\prime}(\xi)\right] .
$$

Again we see that only products of $F$ and $G$ contribute.

\section{Solution for reflection from a uniform slope}

We now introduce a uniform slope $z=\gamma x$, at which we require $\psi_{x}+\gamma \psi_{z}=0$ (i.e. $w=\gamma u$ ). In the following sections we derive the solution for internal-wave reflection from the slope. The procedure is straightforward: we use the general solution obtained at first and second order, viz. Eqs. (19), (25) and (30), and impose the boundary condition at each order. 


\subsection{Order $\epsilon$; primary wave}

We use the general solution (19), and require $\Psi=0$ at the slope (thereby satisfying the boundary condition). This provides a coupling between $F$ and $G$ :

$F\left(\left[\mu_{+}-\gamma\right] x\right)+G\left(\left[\mu_{-}-\gamma\right] x\right)=0$ for all $x$,

as is natural since one of them ( $G$, say) now results from a reflection of the other $(F)$. Hence, for all $\eta$,

$G(\eta)=-F(\lambda \eta)$,

with

$\lambda=\frac{\mu_{+}-\gamma}{\mu_{-}-\gamma}$.

(Critical reflection occurs if $\lambda \rightarrow \infty$, i.e. if the angle of the reflected wave coincides with that of the slope: $\mu_{-}=\gamma$.) Without loss of generality, we may write $F$ as

$F(\xi)=\int_{0}^{\infty} d k a(k) e^{i k \xi}$.

As pointed out by Tabaei et al. (2005), it is crucial that $k$ be either exclusively positive or exclusively negative, since otherwise $F$ cannot represent a purely incident wave. (We can ensure later that $F(\xi)$ actually does describe an incident wave by giving the frequency $\sigma$ the appropriate sign.) The reflected wave is then represented by $G$ in Eq. (32), so the total solution (19) becomes

$\Psi=F(\xi)+G(\eta)=\int_{0}^{\infty} d k a(k)\left[e^{i k \xi}-e^{i k \lambda \eta}\right]$.

Hereafter it will be understood that integrals are taken from zero to infinity without explicitly stating so.

\subsection{Order $\epsilon^{2}$; mean field}

From Eq. (25), with Eqs. (34) and (32), we obtain:

$$
\Psi_{0}=-\frac{2 \lambda}{\sigma}\left(\mu_{+}-\mu_{-}\right) \iint d k d k^{\prime} a(k) a\left(k^{\prime}\right) k k^{\prime} \sin \left(k \xi-k^{\prime} \lambda \eta\right) \text {. }
$$

The boundary condition is automatically satisfied. (This can be seen by splitting the sine into the sum of its two halves, and interchanging $k$ and $k^{\prime}$ in the second term; one then finds that the two terms cancel at the slope.)

\subsection{Order $\epsilon^{2}$; second harmonic}

The right-hand side Eq. (31) can now be written

$$
\begin{aligned}
& J\left(\nabla^{2} \Psi, \Psi\right)= \\
& \lambda\left(\mu_{+}-\mu_{-}\right) \iint d k d k^{\prime} a(k) a\left(k^{\prime}\right) k k^{\prime}\left[\left(1+\mu_{+}^{2}\right) k^{2}-\left(1+\mu_{-}^{2}\right) k^{2} \lambda^{2}\right] e^{i\left(k \xi+k^{\prime} \lambda \eta\right) .}
\end{aligned}
$$

Hence a particular solution to Eq. (29) is

$$
\Psi_{2, p}=3 i \sigma \lambda\left(\mu_{+}-\mu_{-}\right) \iint d k d k^{\prime} S\left(k, k^{\prime}\right) e^{i\left(k \xi+k^{\prime} \lambda \eta\right)},
$$

with

$$
\begin{aligned}
& S\left(k, k^{\prime}\right)= \\
& -\frac{a(k) a\left(k^{\prime}\right) k k^{\prime}\left[\left(1+\mu_{+}^{2}\right) k^{2}-\left(1+\mu_{-}^{2}\right) k^{\prime 2} \lambda^{2}\right]}{A_{2}\left(k \mu_{+}+k^{\prime} \lambda \mu_{-}\right)^{2}-2 B\left(k \mu_{+}+k^{\prime} \lambda \mu_{-}\right)\left(k+k^{\prime} \lambda\right)+C_{2}\left(k+k^{\prime} \lambda\right)^{2}} .
\end{aligned}
$$

However, Eq. (37) does not by itself satisfy the boundary condition at the slope. We must add a solution of the homogeneous part of Eq. (29), which is given by $F_{2}+G_{2}$ in Eq. (30). Moreover, it must be associated with the reflected wave, so we take $F_{2}=0$, and select the appropriate form of $G_{2}\left(\eta_{2}\right)$. We thus arrive at

$$
\Psi_{2}=3 i \sigma \lambda\left(\mu_{+}-\mu_{-}\right) \iint d k d k^{\prime} S\left(k, k^{\prime}\right)\left[e^{i\left(k \xi+k^{\prime} \lambda \eta\right)}-e^{i R\left(k, k^{\prime}\right) \eta_{2}}\right],
$$

where $\eta_{2}$ is defined in Eq. (14), and

$$
R\left(k, k^{\prime}\right)=\frac{\mu_{+}-\gamma}{\mu_{2,-}-\gamma}\left(k+k^{\prime}\right) \text {. }
$$

\subsection{Expressions for single plane waves}

The simplest choice for $a(k)$ is a $\delta$-distribution, $a(k)=\delta(k-$ $k_{0}$ ), so that the incident wave becomes $F(\xi)=\exp i k_{0} \xi$, to which we refer as a single plane wave. The complete lowestorder solution (35), representing the superposition of the incident and reflected primary waves, now becomes

$\Psi=e^{i k_{0} \xi}-e^{i k_{0} \lambda \eta}$.

At the following order, the mean field (36) and second harmonic (39) are given by

$$
\begin{aligned}
& \Psi_{0}=-\frac{2 \lambda}{\sigma}\left(\mu_{+}-\mu_{-}\right) k_{0}^{2} \sin k_{0}(\xi-\lambda \eta) \\
& \Psi_{2}=3 i \sigma \lambda\left(\mu_{+}-\mu_{-}\right) S\left(k_{0}, k_{0}\right)\left[e^{i k_{0}(\xi+\lambda \eta)}-e^{i R\left(k_{0}, k_{0}\right) \eta_{2}}\right] .
\end{aligned}
$$

with

$$
\begin{aligned}
& S\left(k_{0}, k_{0}\right)= \\
& -k_{0}^{2} \frac{\left(1+\mu_{+}^{2}\right)-\left(1+\mu_{-}^{2}\right) \lambda^{2}}{A_{2}\left(\mu_{+}+\lambda \mu_{-}\right)^{2}-2 B\left(\mu_{+}+\lambda \mu_{-}\right)(1+\lambda)+C_{2}(1+\lambda)^{2}} \\
& R\left(k_{0}, k_{0}\right)=2 k_{0} \frac{\mu_{+}-\gamma}{\mu_{2,-}-\gamma} .
\end{aligned}
$$

Note that the denominator in Eq. (43) may vanish for certain parameter values, in which case the second harmonic is resonantly forced (see Sect. 6.3.1).

\section{Analysis of reflection}

We will consider two cases: (i) a single plane wave, for which the expressions are given in Sect. 5.4, and (ii) a "beam", which arises if one takes for example a Gaussian profile for $a(k)$, thus creating a plane-wave packet whose amplitude falls off in the transverse direction. In the latter case, the integral expressions in Eqs. (36) and (39) have to be solved numerically, in general. 


\subsection{Special case: horizontal bottom}

The second harmonic is generated by the forcing term on the righthand side of Eq. (31), in which the reflected wave, $G$, is given by Eq. (32). Here we consider the special case of reflection from a horizontal bottom, i.e. $\gamma=0$. Under the Traditional Approximation, we have $\mu_{+}=-\mu_{-} \equiv \mu$ (i.e. the characteristics are equally steep). With $\gamma=0$, this gives $\lambda=-1$ in Eq. (33), and the forcing term in Eq. (31) reduces to

$$
-2 \mu\left(1+\mu^{2}\right)\left[F^{\prime \prime \prime}(\xi) G^{\prime}(\eta)-G^{\prime \prime \prime}(\eta) F^{\prime}(\xi)\right],
$$

with $G(\eta)=-F(-\eta)$. This term does not, in general, vanish. For example, if we take a Gaussian beam $F=\exp \left(-\xi^{2}\right)$ we obtain

$$
128 \mu^{2}\left(1+\mu^{2}\right) x z\left(\mu^{2} x^{2}-z^{2}\right) \exp \left\{-2\left(\mu^{2} x^{2}+z^{2}\right)\right\} \text {. }
$$

(From this expression the localized character of the forcing is evident; it acts only where the incident and reflected beams interact.) Accordingly, Tabaei et al. (2005) find a non-zero second harmonic for reflection from a horizontal bottom (see their Fig. 11). Furthermore, numerical experiments on internal tides too reveal the generation of higher harmonics for such a reflection (Gerkema et al., 2006a).

The situation is altogether different for a single plane wave, $F=\exp i k_{0} \xi$, in which case (45) vanishes and no second harmonic is generated, as was pointed out already by Thorpe (1987). This can also be seen directly from Eq. (43), where we find $S=0$ if $\mu_{+}^{2}=\mu_{-}^{2}$ and $\lambda=-1$ (Traditional Approximation, horizontal bottom). Thorpe calls $\gamma=0$ a "singular case"; indeed, his results show that for an arbitrarily weak but nonzero inclination (i.e. $0<\gamma \ll 1$ ) one may even find resonant forcing of second harmonics for certain wave frequencies, sharply contrasting with the total absence of forcing for $\gamma=0$.

However, this result hangs on the Traditional Approximation: if we abandon the approximation, we have $\mu_{+} \neq-\mu_{-}$ (i.e. the characteristics associated with the incident and reflected waves are not equally steep). Imposing a horizontal bottom $\left(\gamma=0, \lambda=\mu_{+} / \mu_{-}\right)$now no longer gives a vanishing $S$ in Eq. (43). In other words, higher harmonics are now generated if a single plane wave reflects from a horizontal bottom; the non-traditional terms remove the singular behaviour associated with a horizontal bottom.

\subsection{Examples of beams}

We show examples of beams, obtained by numerical evaluation of the integrals (35), (36) and (39), with $a(k)=1$ in a certain range $\left(k_{1}, k_{2}\right)$, and zero elsewhere. To properly evaluate the integrals in Eq. (39), care must be taken that $S$ contain no singularities. This can be verified numerically, and if necessary the domain of integration $\left(k_{1}, k_{2}\right)$ can be adjusted such that the denominator of $S$ changes its sign nowhere.

For super-inertial waves (i.e. $\sigma>|f|$ ), Fig. 1 shows examples of the primary wave, mean field and second harmonic,
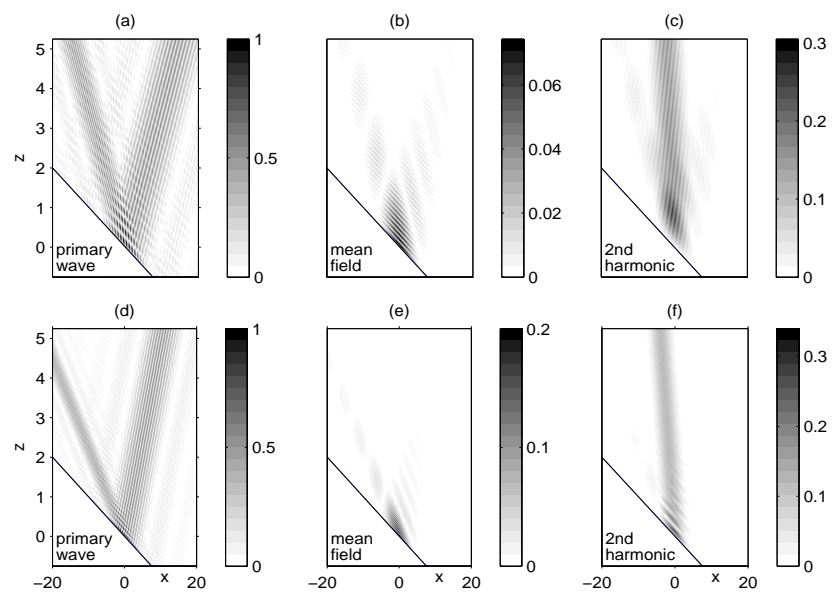

Fig. 1. Reflection of a super-inertial beam from a sloping bottom; the incident wave enters from the upper right corner in (a) and (d). Parameter values are $\gamma=-0.1$ (slope), $N=3 \times 10^{-4} \mathrm{rad} / \mathrm{s}$ (stratification), $\phi=45^{\circ} \mathrm{N}$ (latitude), $\nu=\pi / 2$ (i.e. meridional propagation), $\sigma=1.4 \times 10^{-4} \mathrm{rad} / \mathrm{s}$ (semidiurnal tidal frequency), and $k 1=8$, $k_{2}=10 \mathrm{~km}^{-1}$. Panels (a)-(c) show the solution under the Traditional Approximation (i.e. $f_{s}=0$ ); panels (d)-(f) show the nontraditional results (i.e. $f_{S}$ included). Panels (a) and (d) show $\epsilon|\Psi|$; (b) and (e), $\epsilon^{2}\left|\Psi_{0}\right|$; (c) and (f), $\epsilon^{2}\left|\Psi_{2}\right|$.

calculated with and without the Traditional Approximation (upper and lower panels, respectively). In panels (a) and (d), the incident primary wave enters from the right. In (d), the reflected beam is less steep than the incident one, an immediate consequence of the fact that $\left|\mu_{-}\right|<\left|\mu_{+}\right|$, unlike under the Traditional Approximation (panel (a)), where the two are equal. Furthermore, the second harmonic in (f) is less steep than that in (c); the effect of $f_{s}$ is to decrease $\left|\mu_{2,-}\right|$. The intensity of the interaction is also changed by $f_{s}$, as is clearly visible when one compares the mean fields. Due the relatively short wavelengths involved, the figures give an (optical) illusion suggesting variations along the beam; a zoom of the beam (not shown) demonstrates however that lines of equal phase follow the characteristics, i.e. are aligned to the beam, as they should.

The frequency range of internal waves is enlarged by the non-traditional terms (LeBlond and Mysak, 1978; Gerkema and Shrira, 2005), allowing waves to exist also for slightly sub-inertial frequencies $(\sigma<|f|)$; moreover, the group velocity remains finite at the inertial frequency $\sigma=|f|$. This means there are now two cases of reflection that do not exist under the Traditional Approximation. They are shown in Fig. 2 (upper panels, inertial frequency; lower panels, sub-inertial). In panel (a), the inertial wave enters as a steep beam (associated with $\xi$ ), while the reflected beam is purely horizontal $(\eta)$; the second harmonic $\left(\eta_{2}\right)$ is super-inertial, and directed leftward. In the sub-inertial case (panel d), the reflected beam is also less steep than the incident one, and the second harmonic is again super-inertial (panel f). In panels (c) and (f) one clearly 


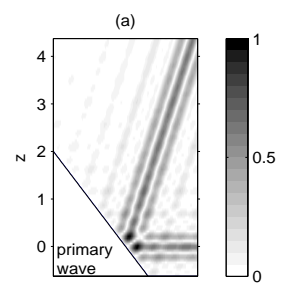

(d)

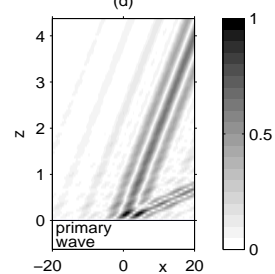

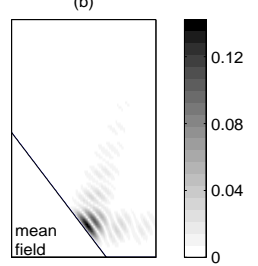

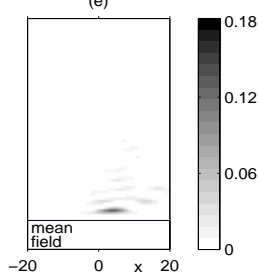

(c)
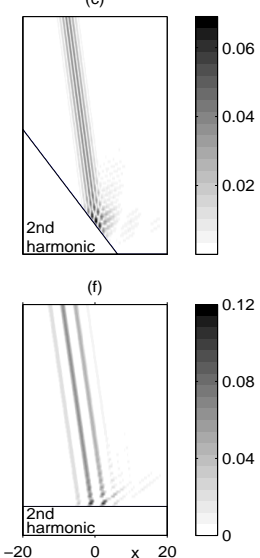

Fig. 2. Reflection of inertial and sub-inertial beams (upper and lower panels, respectively), both non-traditional. Parameters $N, \phi$ and $v$ as in the previous figure; $k 1=2, k_{2}=6 \mathrm{~km}^{-1}$. In the upper panels $\gamma=-0.1$ and $\sigma=f$; in the lower panels $\gamma=0$ and $\sigma=0.97 f$. In (a) and (d), the incident beam enters the domain from the upper right corner.

sees a signal transverse to the principal beam in the region of interaction; this is because the second harmonic contains the particular solution (37), which involves both characteristic coordinates (i.e. $\xi$ and $\eta$ ) and produces a signal in the region of interaction. The free second harmonic, on the other hand, involves only one coordinate $\left(\eta_{2}\right)$.

\subsection{Further analysis of single plane waves}

We recall the expressions for a single plane wave given in Sect. 5.4. They are used in the remainder of this section, to analyse various dependences.

\subsubsection{Resonant forcing}

Following Thorpe (1987), we consider the loci of points, in the slope vs. wave frequency plane, at which the second harmonic is resonantly forced, i.e. where the denominator of Eq. (43) vanishes. The difference from Thorpe (1987) is that we here include non-traditional effects.

Under the Traditional Approximation, the frequency range for the primary wave (i.e. at order $\epsilon$ ) is given by $|f|<\sigma<N$. For the second harmonic (whose frequency is $2 \sigma$ ) to propagate as a free wave, it must similarly satisfy $|f|<2 \sigma<N$, hence $|f| / 2<\sigma<N / 2$. The two requirements together give $|f|<\sigma<N / 2$ for the range of frequencies in which a propagating second harmonic can be generated.

By the same reasoning, one finds with non-traditional effects included a range $\sigma_{\min }<\sigma<\sigma_{\max }$ for the primary wave, and possible forcing of propagating second harmonics in the range $\sigma_{\min }<\sigma<\sigma_{\max } / 2$. Here $\sigma_{\min }$ and $\sigma_{\max }$ are given by (see, e.g. Gerkema and Shrira, 2005).

$\sigma_{\min , \max }=$

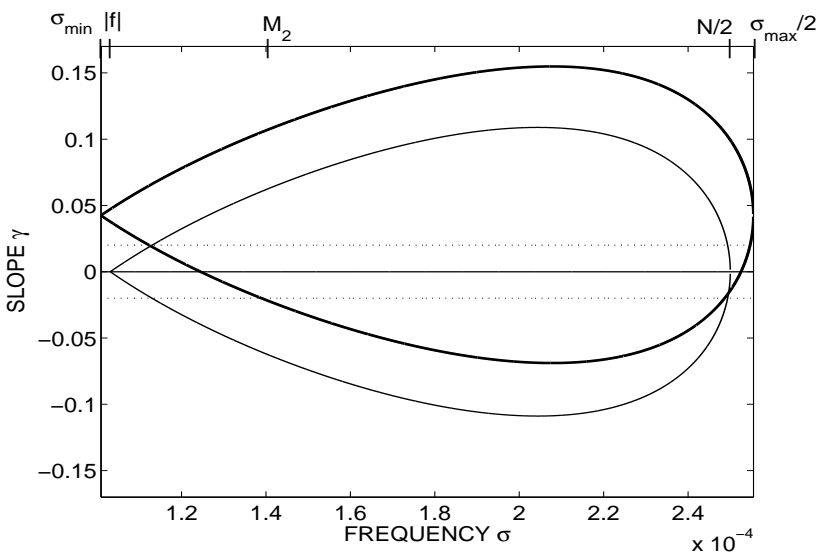

Fig. 3. The loci at which the second harmonic is resonantly forced. Thin line: under the Traditional Approximation; thick line: nontraditional. Horizontal dashed lines indicate the range of values covering $67 \%$ of the slopes in the ocean. Parameter values are: $N=5 \times 10^{-4} \mathrm{rad} / \mathrm{s}$ (a value typical of the deep ocean), latitude $\phi=45^{\circ} \mathrm{N}$ and $\nu=\pi / 2$ (propagation in the meridional direction).

$\frac{1}{\sqrt{2}}\left(\left[N^{2}+f^{2}+f_{s}^{2}\right] \mp\left\{\left[N^{2}+f^{2}+f_{s}^{2}\right]^{2}-(2 f N)^{2}\right\}^{1 / 2}\right)^{1 / 2}$.

An example of the loci is shown in Fig. 3. As discussed in Sect. 6.1, the horizontal bottom (i.e. $\gamma=0$ ) forms a singular case under the Traditional Approximation in that no harmonics are generated at all; the point at $\gamma=0$ is thus excluded from resonant generation. This is no longer the case if the Traditional Approximation is abandoned; then resonant forcing occurs even for reflection from a horizontal bottom.

Another difference is the anisotropy in the horizontal plane created by non-traditional effects, here visible as a $\gamma \leftrightarrow-\gamma$ asymmetry. In the non-traditional case, resonance at nearinertial frequencies $(\sigma \approx|f|)$ requires relatively steep slopes $\sim 0.04$ (Fig. 3, thick line). Slopes in the deep ocean are however predominantly less than 0.02 (Gerkema and Shrira, 2006), as indicated by horizontal dashed lines in Fig. 3. This range of slopes is covered rather by higher frequencies, up to the semi-diurnal tidal frequency $\left(M_{2}\right)$. Under the Traditional Approximation, it is the other way round: the range of abundant slopes here coincides with slightly super-inertial frequencies $(\sigma \lesssim|f|)$. In this respect, it is interesting to note that a marked contrast is found in oceanic internal-wave spectra such as by van Haren et al. (2002): while higher harmonics of the semi-diurnal internal tide are clearly present, none is seen of near-inertial frequencies, even though the latter too contains much energy. The above finding may provide a partial but perhaps not a full explanation of this fact, since the strength of non-traditional effects depends on the horizontal direction of wave propagation $(v)$, and for propagation in the west-east direction, the result would be the same as under the Traditional Approximation.

We finally note that the effect of changing $N$ is straightforward: for larger $N$ the difference between the traditional 


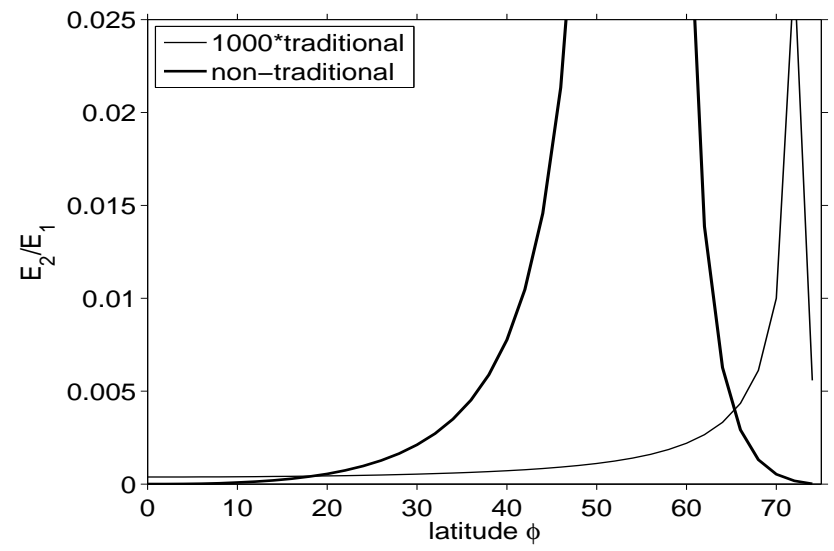

Fig. 4. The ratio of energies of the second harmonic and the incident primary waves, as a function of latitude. Thin line: under the Traditional Approximation; thick line: non-traditional. For better visibility, the thin line shows the ratio artificially multiplied by 1000 . Parameter values are: $\sigma=1.4 \times 10^{-4} \mathrm{rad} / \mathrm{s}, N=5 \times 10^{-4} \mathrm{rad} / \mathrm{s}, \nu=\pi / 2$ (meridional propagation) and slope $\gamma=0.001$. The solution for single plane waves from Sect. 5.4 is used, with $k_{0}=1 \mathrm{~m}^{-1}$ and $\epsilon=10^{-5}$.

and non-traditional curves becomes smaller; strong stratification generally suppresses vertical movements and hence nontraditional effects, since the latter are associated with vertical velocity or vertical acceleration, see Eqs. (1)-(3). (We note, however, that the Traditional Approximation forms a singular limit, as is discussed by Gerkema and Shrira, 2005, implying that some effects may remain present even for strong stratification.)

\subsubsection{Non-resonant forcing}

In this section we consider the energy ratio $E_{2} / E_{1}$ of the second harmonic $\left(E_{2}\right)$ to that of the incident primary wave $\left(E_{1}\right)$, as a function of various parameters. Energy density is defined as

$E=\frac{1}{2} \rho_{*}\left(u^{2}+v^{2}+w^{2}+b^{2} / N^{2}\right)$.

The components $u$ and $w$ follow directly from the streamfunction, while $v$ and $b$ are obtained from Eqs. (16), (17) at lowest order, and from Eqs. (27), (28), but without the Jacobians, for the second harmonic. Only part of the streamfunction is to be used: at lowest order, $F(\xi)$ (the incident wave), and at second order, $G_{2}\left(\eta_{2}\right)$ (the freely propagating part of the second harmonic).

First we consider the dependence on latitude (Fig. 4), for semidiurnal internal tides (M2 frequency). We choose a very mild near-zero slope $(\gamma=0.001)$, but not exactly zero since otherwise no meaningful comparison would be possible between the traditional and non-traditional cases (see Sect. 6.1). In Fig. 4, resonant forcing occurs at $55^{\circ} \mathrm{N}$ (non-traditional) and at $73^{\circ} \mathrm{N}$ (traditional). We note that in the limit $\gamma \rightarrow 0$, res-

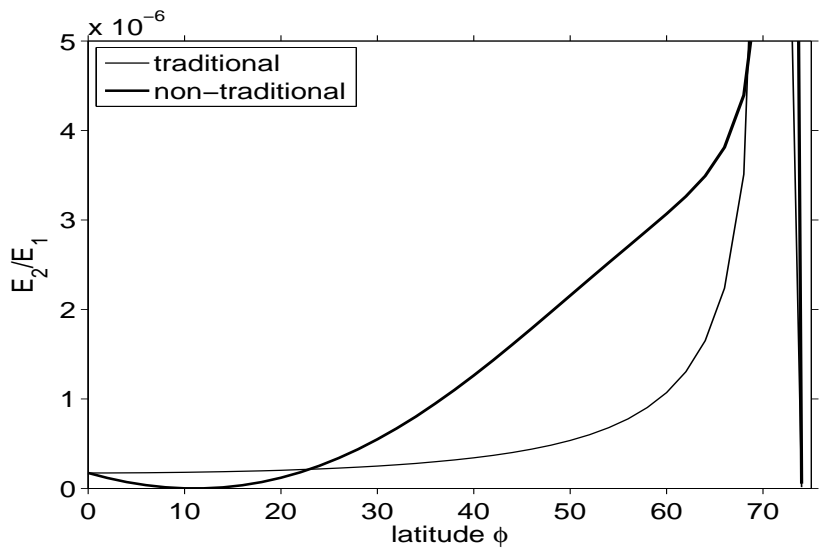

Fig. 5. Parameters as in Fig. 4, but now with stronger stratification: $N=2 \times 10^{-3} \mathrm{rad} / \mathrm{s}$.

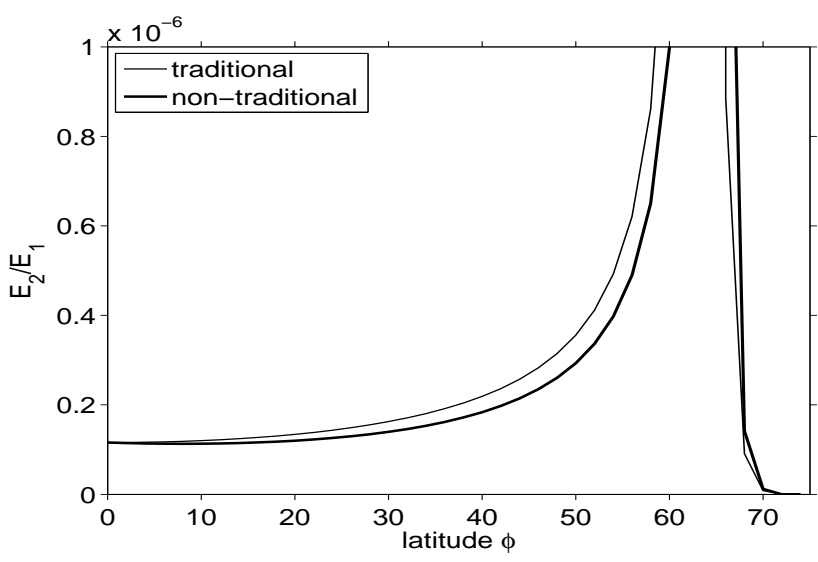

Fig. 6. Parameters as in Fig. 5, but with a still stronger stratification: $N=1 \times 10^{-2} \mathrm{rad} / \mathrm{s}$, a value representative of the seasonal thermocline.

onant forcing occurs precisely at the inertial latitude $74.5^{\circ} \mathrm{N}$ (i.e. where $\sigma=|f|$ ) under the Traditional Approximation, as is easily seen from inspection of the denominator in Eq. (43). Notice that the traditional ratio $E_{2} / E_{1}$ has been artificially multiplied by a factor 1000 for better visibility; its real value is thus much smaller than the non-traditional one. This reinforces the conclusion from Sect. 6.3.1 that non-traditional effects are favourable to generation of higher harmonics at the tidal frequency.

For comparison, we show two plots for the same parameters as in Fig. 4, except that stratification is taken stronger. The traditional signal is now not multiplied by an artificial factor. The curves are indeed closer, but still clearly different in Fig. 5, where $N=2 \times 10^{-3} \mathrm{rad} / \mathrm{s}$. It is only for high values of $N$, such as $N=1 \times 10^{-2} \mathrm{rad} / \mathrm{s}$ in Fig. 6 (a value representative of the seasonal thermocline), that the traditional and non-traditional curves become very similar. By comparing Figs. 4, 5 and 6, it is clear that the intensity of the 


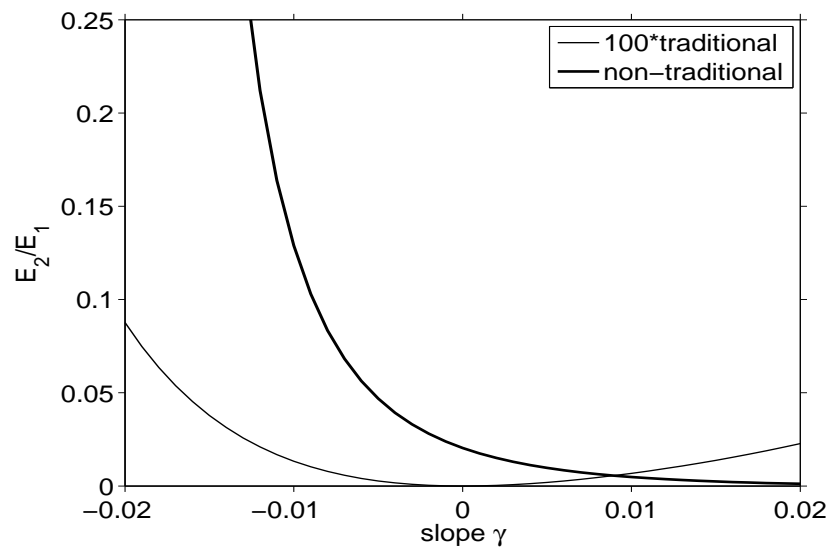

Fig. 7. The ratio of energies of the second harmonic and the incident primary waves, as a function of slope $\gamma$. Thin line: under the Traditional Approximation; thick line: non-traditional. For better visibility, the thin line show the ratio artificially multiplied by 100. Parameter values are: $\sigma=1.4 \times 10^{-4} \mathrm{rad} / \mathrm{s}$ (semidiurnal tidal frequency), $N=5 \times 10^{-4} \mathrm{rad} / \mathrm{s}, \phi=45^{\circ} \mathrm{N}, \nu=\pi / 2$ (meridional propagation). The solution for single plane waves from Sect. 5.4 is used, with $k_{0}=1 \mathrm{~m}^{-1}$ and $\epsilon=10^{-5}$.

second harmonic decreases with increasing $N$ (note the different scalings used on the vertical axes).

In the traditional case (thin line) in Fig. 5, there is only a weak variation of $E_{2} / E_{1}$ with latitude below mid-latitudes. This is in agreement with results from numerical experiments on internal tides (Gerkema et al., 2006b), where the same stratification was used, although there is some ambiguity in their interpretation, partly due the unresolved issue of whether the attainable horizontal resolution is sufficient (Gerkema et al., 2006a,b). Further ambiguities arise from the fact that the generation of the internal tide is itself affected by the Coriolis parameter: the intensity and width of the beam change, not only its angle of propagation, so it is not obvious how a "proper" evaluation of the Coriolis effect can be made. Notice in particular that the ratio $E_{2} / E_{1}$ depends strongly on wavenumber $k_{0}$ because of the quadratic dependence in $S$, see Eq. (43). In any case, the results from Fig. 5 suggest that a much stronger dependence on latitude would be found if non-traditional effects were included. (The terms with $\tilde{f}$ can in fact be easily included in internal-tide generation studies, as was noticed by Baines and Miles, 2000.)

Finally, we consider the dependence of $E_{2} / E_{1}$ on slope steepness $\gamma$, for semi-diurnal tides (Fig. 7), and for nearinertial waves (Fig. 8). The range of prevailing (67\%) values of $\gamma$ is shown. Parameters are identical in both figures, except for the wave-frequency $\sigma$. The first thing to note is that the second harmonic is generally much stronger for tidal than for near-inertial frequencies, by about one order of magnitude. Moreover, the intensity is generally higher if non-traditional effects are included (thick lines), except in the immediate vicinity of resonant peaks in the traditional case.

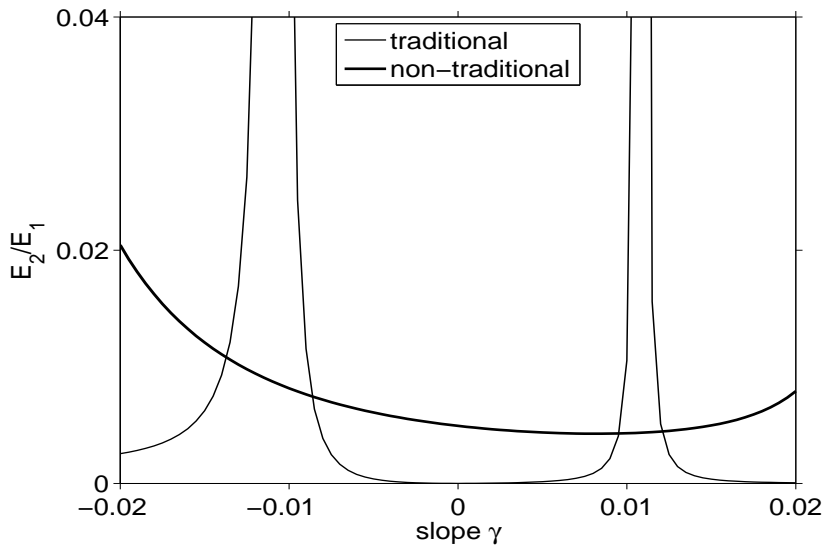

Fig. 8. As in Fig. 7, but now for near-inertial waves, $\sigma=1.05 f=1.083 \times 10^{-4} \mathrm{rad} / \mathrm{s}$. Here the traditional result (thin line) shows the proper outcome (i.e. no artificial multiplication factor is used).

Note that there is now an asymmetry of $\gamma \leftrightarrow-\gamma$ even under the Traditional Approximation, in contrast to the symmetry in the loci for resonant forcing in Fig. 3 (thin line). This means that for a given incident wave and given slope steepness, the intensity of the second harmonic generally depends on the slope orientation, while the value of $|\gamma|$ at which resonance occurs does not. The origin lies in Eq. (43): under the Traditional Approximation one has $\mu_{+}=-\mu_{-} \equiv \mu$ by Eq. (13), and hence $\lambda=(\gamma-\mu) /(\gamma+\mu)$ by Eq. (33), implying $\lambda(-\gamma)=1 / \lambda(\gamma)$. The expression for $S$ reduces to

$$
S\left(k_{0}, k_{0}\right)=-k_{0}^{2}\left(1+\mu^{2}\right) \frac{1-\lambda^{2}}{A_{2} \mu^{2}(1-\lambda)^{2}+C_{2}(1+\lambda)^{2}}
$$

(Traditional Approximation).

Now, if the denominator vanishes for a certain $\lambda=\lambda_{c}$, it vanishes also for $\lambda=1 / \lambda_{c}$ (as can be seen by multiplying the denominator by $\lambda_{c}^{2}$ ). This explains the symmetry with respect to $\gamma \leftrightarrow-\gamma$ in the thin resonance curve in Fig. 3. However, the expression for $S$ as a whole changes if $\lambda$ is replaced by $1 / \lambda$, which explains the asymmetry in the thin lines in Figs. 7, 8.

\section{Discussion}

In the preceding sections we have generalized the theoretical work of Tabaei et al. (2005) on internal-wave reflection and the generation of higher harmonics; we included Coriolis effects, and in particular also the non-traditional effects associated with the horizontal component of the earth rotation vector. The derivation and resulting expressions show that the theory does not become fundamentally more complicated by this generalization; in other words, there is no practical need here to ignore Coriolis effects or make the Traditional Approximation. 
Non-traditional effects were found to bring about two major changes. First, if one considers single plane waves, higher harmonics are generated by reflection from a horizontal bottom, in contrast to the traditional case, where no generation takes place at all (Sect. 6.1). The singularity associated with the horizontal bottom (Thorpe, 1987) is thus removed by non-traditional effects. A second change is related to the inertial vs. tidal preference. Under the Traditional Approximation, resonant forcing occurs for near-inertial waves if one considers the range of slope tangents that prevail in the ocean. With non-traditional effects included, however, the favourable frequencies lie higher, near semi-diurnal tides. The latter situation explains better some oceanic internalwave spectra, as discussed in Sect. 6.3.1. Moreover, the intensity of the second harmonic is generally increased by non-traditional effects, and is usually higher for tidal than for near-inertial frequencies (Sect. 6.3.2). It was shown in Figs. 4-6 that, as expected, strong stratification suppresses non-traditional effects; at the same time, these figures illustrate that the values of $N$ required for a near-total suppression are very high, typically those encountered in the seasonal thermocline, which of course forms only a small part of the vertical column in the ocean. This demonstrates that non-traditional effects are important in internal-wave reflection almost everywhere in the ocean.

In the analysis carried out here we assumed (implicitly) that the reflection of the primary wave is not close to critical; otherwise a different kind of expansion should be made, with frictional effects included (Dauxois and Young, 1999). In fact, strong nonlinearities and overturning may then occur, as was shown in laboratory experiments (Dauxois et al., 2004). In the reflection problem considered here, uniformity was assumed in one of the horizontal directions. The full three dimensional problem for general internal-wave forms has, to our knowledge, not yet been dealt with in the literature. However, for single plane waves (i.e. $\exp i[k x+l y+m z+\sigma t])$, the problem was treated by Thorpe (2000), with emphasis on the induced mean flows, while a new non-traditional criterion for critical reflection was recently derived by Gerkema and Shrira (2006).

Acknowledgements. This work is financially supported by the NWO/ALW program CLIMA-DIM. The author thanks V. Shrira and H. van Haren for ongoing discussions on "non-traditional" issues.

Edited by: R. Grimshaw

Reviewed by: two referees

\section{References}

Baines, P. G. and Miles, J. W.: On topographic coupling of surface and internal tides, Deep-Sea Res. I, 47, 2395-2403, 2000.

Dauxois, T. and Young, W. R.: Near-critical reflection of internal waves, J. Fluid Mech., 390, 271-295, 1999.

Dauxois, T., Didier, A., and Falcon, E.: Observation of near-critical reflection of internal waves in a stably stratified fluid, Phys. Fluids, 16, 1936-1941, 2004.

Gerkema, T. and Shrira, V. I.: Near-inertial waves in the ocean: beyond the 'traditional approximation', J. Fluid Mech., 529, 195219, 2005.

Gerkema, T. and Shrira, V. I.: Non-traditional reflection of internal waves from a sloping bottom, and the likelihood of critical reflection, Geophys. Res. Lett., 33, L06611, doi:10.1029/2005GL025627, 2006.

Gerkema, T., Staquet, C., and Bouruet-Aubertot, P.: Non-linear effects in internal-tide beams, and mixing, Ocean Modelling, 12, 302-318, 2006a.

Gerkema, T., Staquet, C., and Bouruet-Aubertot, P.:, Decay of semi-diurnal internal-tide beams due to subharmonic resonance, Geophys. Res. Lett., 33, L08604, doi:10.1029/2005GL025105, 2006b.

Gostiaux, L., Dauxois, T., Didelle, H., Sommeria, J., and Viboux, S.: Quantitative laboratory observations of internal wave reflection on ascending slopes, Phys. Fluids, 18, 056602, 2006.

Lamb, K. G.: Nonlinear interaction among internal wave beams generated by tidal flow over supercritical topography, Geophys. Res. Lett., 31, L09313, doi:10.1029/2003GL019393, 2004.

LeBlond, P. H. and Mysak, L. A.: Waves in the ocean, Elsevier, 1978.

Peacock, T. and Tabaei, A.: Visualization of nonlinear effects in reflecting internal wave beams, Phys. Fluids, 17, 061702, 1-4, 2005.

Tabaei, A., Akylas, T. R., and Lamb, K. G.: Nonlinear effects in reflecting and colliding internal wave beams, J. Fluid Mech., 526 , 217-243, 2005.

Thorpe, S. A.: On the reflection of a train of finite-amplitude internal waves from a uniform slope, J. Fluid Mech., 178, 279-302, 1987.

Thorpe, S. A.: The effects of rotation on the nonlinear reflection of internal waves from a slope, J. Phys. Oceanogr., 30, 1901-1909, 2000 .

van Haren, H., Maas, L. R. M., and van Aken, H.: On the nature of internal wave spectra near a continental slope, Geophys. Res. Lett., 29, 1615, doi:10.1029/2001GL014341, 2002.

Visser, A. W.: On tidal rectification, friction, and geostrophic degeneracy, J. Phys. Oceanogr., 24, 2196-2200, 1994. 